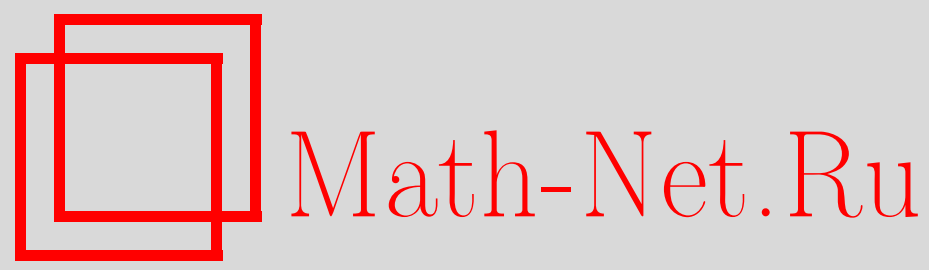

А. В. Домрин, О затягивании максимально комплексных циклов CR-подмногообразиями, $M a-$ тем. заметки, 1996, том 60, выпуск 5, 776-777

DOI: https://doi.org/10.4213/mzm1891

Использование Общероссийского математического портала MathNet.Ru подразумевает, что вы прочитали и согласны с пользовательским соглашением

http://www . mathnet.ru/rus/agreement

Параметры загрузки:

IP : 54.198 .67 .100

26 апреля 2023 г., 18:10:15

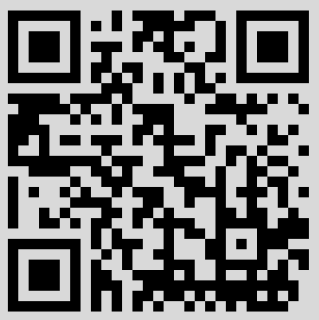




\section{О ЗАТЯГИВАНИИ МАКСИМАЛЬНО КОМПЛЕКСНЫХ ЦИКЛОВ CR-ПОДМНОГООБРАЗИЯМИ}

\section{А. В. Домрин}

Хорошо известно, что в $\mathbb{C}^{2}$ существует вполне вещественный диск, граница которого совпадает с единичной окружностью $\left\{\left(w_{1}, w_{2}\right) \in \mathbb{C}^{2}|| w_{1} \mid=1, w_{2}=0\right\}$. В качестве такого диска можно взять, например, граффик $\left\{(z, g(z)) \in \mathbb{C}^{2}|| z \mid \leqslant 1\right\}$ функции $g(z)=(1-z \bar{z}) \bar{z}(1+i z \bar{z})$. В связи с теоремой единственности для CR-функций недавно был поставлен вопрос о существовании подобных примеров в $\mathbb{C}^{m+1}$, $m \geqslant 2$. А именно, существует ли диффеоморфное замкнутому $2 m$-мерному шару CR-подмногообразие $(\mathrm{CR}$-размерности $m-1)$ в $\mathbb{C}^{m+1}$, граница которого совпадала бы с единичной сферой

$$
\left\{\left.\left(w_{1}, \ldots, w_{m+1}\right) \in \mathbb{C}^{m+1}|| w_{1}\right|^{2}+\cdots+\left|w_{m}\right|^{2}=1, w_{m+1}=0\right\} ?
$$

Основная цель настоящей заметки - дать (отрицателный) ответ на этот вопрос. Фактически будет доказано следующее более общее утверждение (где, как обычно, $B^{k}$ и $S^{k}$ обозначают $k$-мерный замкнутый шар и $k$-мерную сферу).

УтВеРЖДЕНИЕ 1 . Пусть $m \geqslant 2$. Пусть $M^{2 m} \subset \mathbb{C}^{m+1}$ есть $2 m$-мерное

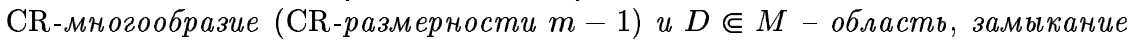
$\bar{D}$ которой диффеоморфно $B^{2 m}$. Тогда $(2 m-1)$-мерное подмногообразие $\partial D \subset \mathbb{C}^{m+1}$ не является максимально комплексным.

Напомним, что гладкое $(2 m-1)$-мерное многообразие $\Gamma \subset \mathbb{C}^{m+1}$ называется максимально комплексным, если комплексная касательная плоскость $T_{p}^{c} \Gamma=$ $T_{p} \Gamma \cap i T_{p} \Gamma$ имеет комплексную размерность $m-1$ в каждой точке $p \in \Gamma$. Ясно, что если $\Gamma$ лежит на некоторой комплексной гиперповерхности в $\mathbb{C}^{m+1}$, то $\Gamma$ максимально комплексно. Таким образом, утверждение 1 дает отрицательньй ответ на поставленный вьше вопрос.

ДоКАЗАТЕЛЬСТВо УТВЕРЖ ДЕНИЯ 1 . Допустим, что подмногообразие $\partial D$ максимально комплексно. Тогда $T_{p}^{c} M=T_{p}^{c} \partial D$ для всех $p \in \partial D$. Обозначим через $n_{p}$ единичньй вектор внешней нормали к $\partial D$ (как подмногообразию $M$ ) в точке $p \in \partial D$, а через $E$ - расслоение со слоем $S^{1}$ над $M$, слоем которого в точке $x \in M$ служит множество всех векторов единичной длины в пространстве $T_{x} M / T_{x}^{c} M$. Расслоение $E$ тривиально над $\bar{D}$ и $p \mapsto n_{p}$ есть его сечение над $\partial D$. Поскольку при $m \geqslant 2$ имеем $\pi_{2 m-1}\left(S^{1}\right)=0$, это сечение гладко продолжается на $\bar{D}$. Тем самым, получаем всюду отличное от 0 векторное поле на замкнутом $2 m$-мерном шаре $B^{2 m} \subset \mathbb{R}^{2 m}$ (диффеоморфном $\bar{D}$ ), которое направлено наружу на границе. Но, как хорошо известно (см., например, $[1, \S 6$, лемма 3$]$ ), такого поля не существует. Полученное противоречие доказывает утверждение 1.

Следующее утверждение показывает, что топологически неэквивалентная шару область на $\mathrm{CR}$-многообразии $M^{2 m} \subset \mathbb{C}^{m+1}$ может быть ограничена максимальн комплексным циклом. Мы построим пример с $m=2$.

Работа выполнена при поддержке Российского фонда фундаментальных исследований, грант № 96-01-01218. 
УтВЕРЖДЕнИЕ 2. Существуют CR-многообразие $M^{4} \subset \mathbb{C}^{3}$ (CR-размерности 1) и область $D \Subset M$, замыкание которой диффеоморфно $S^{1} \times B^{3}$, такие, что дD является максимально комплексным подмногообразием в $\mathbb{C}^{3}\left(\right.$ ицффеоморфным $\left.S^{1} \times S^{2}\right)$.

ДоКАЗАТЕЛЬство. Возьмем в качестве $M$ график

$$
\left\{\left(z_{1}, z_{2}, f\left(z_{1}, z_{2}\right)\right) \in \mathbb{C}^{3} \mid\left(z_{1}, z_{2}\right) \in \mathbb{C}^{2}, z_{1} \neq 0\right\}
$$

некоторой функции $f$ вида

$$
f\left(z_{1}, z_{2}\right)=Q\left(\left|z_{1}\right|^{2}\right) \cdot\left(\left(\left|z_{1}\right|^{2}-1\right)^{2}+\left|z_{2}\right|^{2}-\varepsilon^{2}\right)
$$

а в качестве $D$ - часть этого графика, лежащую над областью

$$
\Omega=\left\{\left.\left(z_{1}, z_{2}\right) \in \mathbb{C}^{2}\left|\left(\left|z_{1}\right|^{2}-1\right)^{2}+\right| z_{2}\right|^{2}-\varepsilon^{2}<0\right\}
$$

где $0<\varepsilon<1$. Тогда подмногообразие $\partial D \subset \mathbb{C}^{3}$ лежит в плоскости $z_{3}=0$ и, следовательно, является максимально комплексным. Функцию $Q:(0,+\infty) \rightarrow \mathbb{C}$, входящую в определение $f$, будем считать $C^{\infty}$-гладкой и подберем ее так, что

(i) $Q \neq 0$ на $(0,+\infty)$,

(ii) функция $\gamma(t)=Q(t) \rho(t)$, где $\rho(t)=(t-1)^{2}-\varepsilon^{2}$, удовлетворяет $\gamma^{\prime}(t) \neq 0$ для всех $t \in(0,+\infty)$

(ясно, что такие функции $Q(t)$ сушествуют).

Чтобы проверить, что $M$ есть CR-многообразие, достаточно доказать, что вектор $\left(f_{\overline{z_{1}}}\left(z_{1}, z_{2}\right), f_{\overline{z_{2}}}\left(z_{1}, z_{2}\right)\right)$ отличен от 0 в каждой точке $\left(z_{1}, z_{2}\right) \in \mathbb{C}^{2}$ с $z_{1} \neq 0$. (Здесь $f_{\overline{z_{j}}}$ есть частная производная $f$ по $\overline{z_{j}}$ ). Но $f_{\overline{z_{2}}}\left(z_{1}, z_{2}\right)=Q\left(\left|z_{1}\right|^{2}\right) \cdot z_{2} \neq 0$ всюду, где $z_{2} \neq 0$ (согласно свойству (i) функции $Q(t)$ ), а для $z_{2}=0$ прямое вычисление дает, что $f_{\overline{z_{1}}}\left(z_{1}, 0\right)=z_{1} \cdot \gamma^{\prime}\left(\left|z_{1}\right|^{2}\right)$. Эта величина отлична от 0 при $z_{1} \neq 0$ в силу свойства (ii) функции $Q(t)$.

Наконец, утверждения о том, что $D$ и $\partial D$ дифффеоморфиы $S^{1} \times B^{3}$ и $S^{1} \times S^{2}$ соответственно, вытекают из того, что область $\Omega$ диффеоморфна метрической $\varepsilon$-окрестности окружности $S^{1}=\left\{\left(z_{1}, 0\right) \in \mathbb{C}^{2}|| z_{1} \mid=1\right\}$ в $\mathbb{C}^{2}$.

Московский государственный университет

Поступило

им. М. В. Ломоносова

28.02 .95

E-mail: domrin@mian.su

\section{СПИСОК ЦИТИРОВАННОЙ ЛИТЕРАТУРЫ}

1. Милнор Дж. Топология с дифференциальной точки зрения // Дифференциальная топология. Начальный курс. М.: Мир, 1972. 